\title{
El mundo académico y las políticas públicas frente a la urgencia del desarrollo sustentable en América Latina y el Caribe
}

\author{
Cristian Parker Gumucio \\ Universidad de Santiago de Chile, Santiago, Chile. \\ Email: cristian.parker@usach.cl
}

Resumen: La relación entre el mundo académico y las políticas públicas (PP) en América Latina y el Caribe (ALC) en función de las tareas del autentico desarrollo sustentable adolece de déficits. Este artículo comienza analizando la relación entre PP y las tareas del desarrollo, y las características que adquiere hoy la gobernanza ambiental. A la luz de las transformaciones de la sociedad del conocimiento y las exigencias propias de la complejidad de la ecología y el ambiente se analizan las interacciones entre el mundo de la academia y el mundo de las políticas públicas. Se finaliza proponiendo un nuevo paradigma en la relación entre ambos mundos. Un nuevo pacto entre el mundo de la academia y el de las políticas públicas, debe asumir el objetivo de la transición hacia la sustentabilidad, la integración y cooperación regional, generando un espacio para la democracia ambiental, lo que supone re-pensar la forma cómo se diseña e implementa la propia PP y la gobernanza ambiental. ${ }^{1}$

Palabras clave: Políticas públicas, gobernanza ambiental, desarrollo sustentable, nuevos paradigmas, mundo académico.

\section{The academic world and public policies facing the urgencies of sustainable development in Latin America and the Caribbean}

\begin{abstract}
The relationship between the academic world and public policy (PP) in Latin America and the Caribbean (LAC) facing the tasks of genuine sustainable development suffers from deficits. This article begins by analyzing the relationship between PP and development tasks, and the features acquired by current environmental governance. In the light of the changes of knowledge society and the complexity of ecology and environment, interactions between the world of academia and the world of PP are discussed.

This paper ends proposing a new paradigm in the relationship between these two worlds. A new pact between the world of academia and PP must assume the purpose of the transition to sustainability, regional cooperation and integration, and creating space for environmental democracy. All this means re-thinking the way PP and environmental governance are conceived and implemented.
\end{abstract}

Key words: Public policy, environmental governance, sustainable development, new paradigms, academia. 


\section{O mundo acadêmico e as Políticas públicas de frente à urgência do desenvolvimento sustentável na América Latina e no Caribe}

Resumo: A relação entre a academia e políticas públicas (PP) na América Latina e no Caribe (ALC) em função das tarefas autênticas do desenvolvimento sustentável sofre de déficits. Este artigo começa por analisar a relação entre PP e tarefas de desenvolvimento e as características que hoje adquire a governança ambiental. À luz das mudanças na sociedade do conhecimento e as exigências próprias da complexidade da ecologia e o meio ambiente são discutidas as interações entre o mundo acadêmico e o mundo das políticas públicas. Termino, propondo um novo paradigma na relação entre ambos os mundos. Um novo pacto entre o mundo da academia e das políticas públicas deve assumir a meta da transição para a sustentabilidade, integração e cooperação regional, criando um espaço para a democracia ambiental, o que significa repensar a forma como se desenha e implementa a própria PP e a governança ambiental.

Palavras-chave: Políticas públicas, governança ambiental, desenvolvimento sustentável, novos paradigmas, mundo acadêmico.

$* * *$

La relación entre el mundo académico y las políticas públicas en América Latina y el Caribe (ALC) están siendo cada vez más demandadas por los desafíos que plantea el desarrollo sustentable. Para analizar esta temática necesitamos primero revisar la relación existente entre el mundo de las políticas públicas y las tareas del desarrollo, en el marco de las características que adquieren hoy tanto la Política Pública (PP) como la gobernanza ambiental. De esta manera podemos desarrollar la interacción existente e ideal entre el mundo de la academia y el mundo de las políticas públicas para finalizar proponiendo un nuevo paradigma en su relación colaborativa en la perspectiva de contribuir al desarrollo sustentable.

\section{Sobre los desafíos del desarrollo sustentable para las políticas públicas}

La PP ha sido planteada como la principal acción institucional de carácter público orientada hacia la mejora de las condiciones del bien común en cada sociedad.

Puede definirse a la PP como el sistema de cursos de acción - generalmente administrativos- de los gobiernos y parlamentos enfocados hacia los temas de una agenda publica, definidos por esas entidades como susceptibles de ser regulados, normados, o financiados en el marco legal vigente. (ver Dye 1992; Lahera, 1993, 2002; Podestá, 2001; Ramírez, 2008; Oviedo, 2009; Moreno, 2009).

Entonces reflexionar sobre PP es hacerlo sobre lo que deberá hacer- 
se para cambiar el hoy en pro de un futuro distinto. Esto implica pensar que una de las finalidades primordiales de las políticas es la capacidad de planear y articular de mejor forma el futuro.

En enero de 2013 se realizó la I Cumbre CELAC -UE (Cumbre de Gobiernos de América Latina y el Caribe y la Unión Europea)², que debatió acerca del futuro que debía considerar de manera relevante la sustentabilidad $^{3}$. La temática de esta Cumbre nos recuerda que todo proceso de inversión debe inscribirse en un determinado modelo de desarrollo y por lo mismo la reflexión acerca de la PP nos lleva a reflexionar acerca de los principales desafíos del desarrollo.

Pueden definirse esquemáticamente los principales desafíos que el Desarrollo Sustentable plantea a la PP en ALC en base a las siguientes dimensiones identificadas por el propio Banco Mundial y por CEPAL (ver Vergara, 2009; Banco Mundial 2010; CEPAL, 2012):

a) creciente crisis ambiental con sus manifestaciones en el cambio climático (CC), en la biomasa útil de recursos naturales explotables en declive, y la grave degradación ambiental; b) persistencia de la pobreza y de la inequidad; c) creciente demanda energética y escasez creciente de los recursos hídricos.

Estos desafíos se nos plantean en el marco de los modelos de desarrollo que se han ido implementando y están vigentes en la región. Cualquiera sea la orientación de esos modelos, si apuntan a la persistencia del neoliberalismo o conforman una alternativa neodesarrollista y/o popular, es un hecho que las economías latinoamericanas presentan deficiencias. Los economistas afirman que las debilidades de nuestros países para crecer económicamente residen en variados factores. Entre los principales factores que afectan la competitividad (Sachs y Vial, 2002; Wallace, 2011) frente a los países altamente posicionados en la sociedad del siglo XXI se cuentan:

a) la distribución desigual del ingreso y la pobreza; b) la calidad de la educación que es relativamente baja; c) el bajo nivel de inversión en innovación y tecnologías; d) la insuficiente infraestructura y e) economías demasiado dependientes de la exportación de productos primarios.

Sin duda la reincorporación de las economías latinoamericanas al sistema global desde la década de los 80’ enfatizó un modelo capitalista exportador que, luego del gran impulso de los países emergentes (especialmente China este último tiempo) ha significado una reubicación de estas economías en una nueva división del trabajo internacional asignándoles la tarea de ser economías proveedoras de materias primas. En efecto, desde fines de los 90’ los modelos de desarrollo vigentes en esta región, más allá de las orientaciones políticas de sus gobiernos, se están focalizando cada vez más en una opción extractivista, que significa la re-primarización de las economías ${ }^{4}$. 
En ese contexto la problemática ambiental de hoy tiene dos grandes dimensiones interrelacionadas, por una parte el impacto ambiental de la actividad humana y por otra el uso de los recursos naturales que son escasos. El problema de la civilización actual reside precisamente que ambos aspectos están coincidiendo de tal forma que presenciamos simultáneamente el riesgo de la contaminación y degradación ambiental y el riesgo del agotamiento de ciertos recursos vitales para la vida humana. Nuestra sociedad se ve enfrentada entonces a las consecuencias del impacto ambiental, que involucra temáticas tan decisivas y debatidas como el CC y la biodiversidad, pero es necesario además, a la luz de los procesos de globalización y del crecimiento demográfico, tomar en consideración el riesgo de la disminución de los recursos naturales: el agua, los alimentos, las energías fósiles, todos ellos recursos que son y serán crecientemente demandados por una población mundial en ascenso y con mayor capacidad de demanda.

Son los propios organismos internacionales los que nos han puesto en alerta sobre varios de estos desafíos globales que conciernen a la comunidad internacional y por cierto a Europa y a América Latina y el Caribe (Samaniego, 2009).

Ya el Informe Mundial sobre energía emanado de la Agencia Internacional de Energía, a fines de 2012, es preocupante cuando afirma que: "Teniendo en cuenta todos los nuevos desarrollos y políticas, el mundo está fracasando en conducir el sistema energético mundial por una senda más sostenible”(IEA, 2012: 1).

Pero en el contexto de los alcances del impacto del CC reconocidos por los organismos especializados (IPCC 2007; PNUD, 2011 y PNUMA, 2011) los combustibles fósiles siguen siendo predominantes en el mix energético mundial, apoyados por subvenciones que alcanzaron un valor de \$ 523000 millones de USD en 2011, lo que supone un aumento de casi el 30\% respecto a 2010 y una cantidad seis veces mayor a las subvenciones a las energías renovables. El informa de la AIE agrega: "Las emisiones en el Escenario de Nuevas Políticas corresponden con un aumento de la temperatura media global a largo plazo de 3,6 ${ }^{\circ} \mathrm{C}$.” (IAE, 2012). Esta predicción nos lleva ciertamente a un decurso futuro de grave insostenibilidad. Por lo mismo la propia Agencia Internacional de Energía proponía en junio de 2013 ante la persistencia de este pesimista escenario algunas políticas para mantener el objetivo de los 2c grados en el clima global (IEA, 2013).

Por otra parte, la escasez del recurso hídrico se hace ver en todos los rincones del mundo y la huella de agua que nos permite medir el consumo de este recurso a nivel mundial nos está indicando que su distribución sigue los patrones del desarrollo relativo. Sabemos que el tema del acceso al agua se está haciendo cada vez más problemático en muchos países de ALC y los conflictos por el agua no cesan de producirse en muchas localidades $^{5}$. 
América Latina y el Caribe y Europa no pueden estar indiferentes frente a estos desafíos y no lo han estado como bien lo reflejan sus conclusiones en la Cumbre de Madrid en 2010 acerca del Desarrollo Sustentable, la Energía Limpia, y la Biodiversidad (Consejo de la UE, 2010).

\section{Nuevas formas y desafíos de la Gobernanza Ambiental}

Cuando la PP asume el desafío ambiental debe asumir la complejidad de los escenarios y la multiplicidad de actores involucrados. Se ha llamado gobernanza ambiental a la capacidad de articulación de acuerdos en el marco de procesos de negociación de políticas y opciones de desarrollo entre todos los actores involucrados en el tema ambiental y ya no sólo al Estado y sus organismos.

En gran medida por efectos de la globalización de las últimas décadas, la gobernanza ambiental está adquiriendo nuevas características (Clapp, 1998; Cashore, 2002; Paavola, 2007; Baud et al, 2011) Hay una nueva relación Estado - mercado donde se reconoce a los privados una responsabilidad ambiental que están asumiendo bajo la bandera de la responsabilidad social corporativa; la propia gobernanza ambiental está sometida a la internacionalización (Poole, 2012) dado que tiene una dimensión internacional que supera fronteras y se torna cada vez más decisiva; aparecen en escena una multiplicidad de actores que van más allá del actor estatal y sus organismos de gobierno, los legisladores, los organismos de evaluación y control ambiental, los consultores de política ambiental, las entidades especializadas empresariales, y por cierto los movimientos sociales, indígenas y ambientalistas; la gobernanza ambiental está siendo demandada por la sociedad civil y en todos lados se hace sentir la demanda por una democratización de las decisiones de PP y privada que dicen relación e impactan el medio ambiente (Ford, 2003, Hysing, 2013); los conflictos socioambientales en la totalidad de las naciones latinoamericanas tienden a crecer lo que plantea una presión por formas y mecanismos de gestión de esos conflictos, tema central de gobernanza; está también las tareas de la innovación productiva y tecnológica que desafían a nuevas formas de gobernanza ambiental en términos del problema del conocimiento; finalmente las sociedades se ven confrontadas a asumir las tareas del Milenio que incluyen el desarrollo sustentable con sus exigencias de una gobernanza inclusiva y que genere cambios hacia una sociedad y economía equitativa, verde y justa, todo lo cual le exige a la gobernanza asumir las tareas de una transición de esta envergadura.

En un estudio que se está realizando a nivel conjunto entre equipos europeos y latinoamericanos (Proyecto ENGOV) ${ }^{6}$ sobre gobernanza ambiental en ALC está quedando claramente en evidencia el carácter multidimencional y la complejidad de la gobernanza ambiental.

En efecto, los estudios de los sistemas socio-ecológicos se enfocan hacia el carácter multiescalar, "multi-stakeholder”, del proceso de elabora- 
ción y toma de decisiones ambientales, por lo que el conocimiento debe orientarse hacia los sistemas multinivel de la gobernanza ambiental en el cual diferentes actores desempeñan diferentes niveles de poder, autoridad y acción para determinar "quién recibe qué” y "quién finalmente decide” (Bull, 2010). En tanto las funciones del Estado se han dispersado hacia arriba, hacia abajo y hacia fuera a actores no estatales, una nueva geografía de la gobernanza ha emergido.

El orden de complejidad de los problemas y tareas que asume la gobernanza ambiental en ALC está siendo reconocido a nivel de los encargados de la PP pero no siempre el conjunto de soluciones de tipo institucional, técnico o político son las adecuadas para conducir a procesos que garanticen tanto la participación ciudadana, las exigencias técnicas y económicas de los proyectos, como la garantía de protección ambiental y de orientación sustentable de esos proyectos de desarrollo en los distintos niveles (Parker, 2011).

Frente a las necesidades del desarrollo y sus impactos ambientales, frente a las necesidades de reducir pobreza, desarrollarse equitativamente y sin dañar el medio ambiente, los gobiernos y las sociedades latinoamericanas, tanto como los encargados de la PP y los medios académicos, tienen el desafío de colaborar antes que la problemática ambiental se vea agudizada y los conflictos socioambientales se vean incrementados.

El aporte de la academia a la PP - que ha sido escaso y problemático, como veremos - va a colaborar con la gobernanza ambiental en la medida en que asuma que tiene que poner a la disposición de estos propósitos toda su capacidad, conocimiento y expertice, para avanzar en las tareas desde una gobernanza ambiental de gestión más allá, en el marco de los desafíos planteados por la transición, hacia una gobernanza ambiental de cambio.

\section{El Mundo de las políticas públicas en la sociedad del conocimiento}

En el contexto de los desafíos del desarrollo sustentable y de la gobernanza ambiental reseñados es necesario volver sobre la PP y sus condicionantes. La PP en el siglo XXI ha estado sometida a un conjunto de situaciones que están redefiniendo su carácter y reorientado sus necesidades.

La PP en el contexto de la sociedad de conocimiento está sometida a variados cambios derivados principalmente de la nueva forma de producir conocimiento, de los avances científicos-tecnológicos, de las nuevas tecnologías de las comunicaciones, el transporte y la información, y de los nuevos actores de la PP.

Particularmente interesante resulta anotar que en el ámbito de la 
producción y circulación del conocimiento las sociedades del Siglo XXI han modificado las dinámicas clásicas que la sociedad industrial tenía en la producción de ciencia, tecnología e innovación. Se ha pasado de un acento en la investigación disciplinaria centrada en las definiciones del científico a una investigación interdisciplinaria centrada en problemas; de la ciencia básica hacia un acento en la innovación; de un acento en la ciencia teórica a un acento en la ciencia aplicada; de un acento en la ciencia disciplinaria a un acento en el conocimiento inter y multidisciplinario; de procesos de investigación individuales a procesos multidimensionales y colectivos; de un acento en procesos investigativos en el mundo académico-universitario nacional, a procesos descentralizados y múltiples en variados centros de investigación e innovación internacionales. Se ha pasado del modo uno de hacer ciencia al modo dos. En tanto la producción de conocimientos estaba ubicada en instituciones científicas, y guiada y estructurada por disciplinas científicas; ahora su ubicación, prácticas y principios son mucho más heterogéneos (Gibbons et al, 1994). No todas las áreas del conocimiento han transitado al modo dos ni es este un concepto aceptado universalmente pero el menos es una tipología que indica ciertamente la dirección en la cual evoluciona la producción de la ciencia en el siglo XXI (Hessels y Van Lente, 2010). Una explicación plausible de cómo se ha pasado del modo uno de hacer ciencia centrado en la academia tradicional al modo dos más flexible y dinámico, es por el cambio en la llamada triple hélice (Etzkowitz y Leydesdorff, 2000) esto es en la relación que se ha establecido a fines del siglo XX entre el gobierno, la academia y la industria. Ello explica los procesos de desarrollo tecnológico e innovación y de transferencia de conocimientos actuales.

Todo este cambio en la dinámica y en los modos de producción del conocimiento, están afectando de manera decisiva al modo de producir y usar el conocimiento en la PP y desafían a los ámbitos todavía tradicionales de muchas de las instancias universitarias latinoamericanas.

En cuanto a los nuevos contextos que influyen en el mundo de las PP es interesante anotar que estas ya no constituyen monopolio de los actores estatales, enfrentan a nuevos actores y se ven exigidas por las reformas de la política en la región (Gomáriz, 2007). Después de las décadas del experimento neoliberal las políticas públicas a nivel mundial y en especial a nivel latinoamericano parecen haber recuperado la confianza en el Estado (Arriagada, 2006). Es el giro de lo que se ha llamado una tercera generación de políticas sociales y, en general, de PP. Cambios en el incremento de la densidad de las políticas y una mayor determinación propiamente política, superando, como planteara el BID, el estadio de las políticas tecnocráticas y reinsertando éstas en el funcionamiento de los sistemas políticos.

Estos cambios hacia fines de la primera década del siglo XXI suponen una redefinición del rol del mercado como principio orientador de la sociedad, pero no retornan a las políticas estatales donde el monopolio lo tenía la planificación centralizada. En efecto, es necesario reconocer que si 
bien el mercado es un principio importante de organización de la economía desde el mercado no surge ni puede surgir ninguna PP. Así como tampoco el monopolio del estado genera PP sustentable. Es precisamente a partir de un rol preponderante del estado, pero no con carácter absoluto, que se funda la PP actual, generando instancias de planificación y programación indicativa y regulatorias de la acción de los mercados, pero dejando un marco adecuado a la iniciativa privada, sobre la base de la preponderancia de los principios del bien común.

Pero con la globalización se ha generado una dinámica muy propia de la PP actual que supera los estrechos marcos que anteriormente le imponía el Estado-nación. El peso de las variables externas es cada vez mayor en la política doméstica, estableciendo condicionalidades sobre las decisiones del desarrollo nacional impensables en la lógica del "orden wesfaliano" (Rojas, 2011).

Más allá de las fronteras nacionales la PP hoy está tensionada entre lo global y lo local, y demandada por los procesos de descentralización y los regionalismos. Nadie duda de la relevancia, a veces decisiva, que proviene de las PP que provienen de instancias globales: Banco Mundial, Organismos de Nacional Unidas, CEPAL, etc.

Por otra parte a la PP actual se la impone la necesidad de avanzar en las áreas de la integración especialmente a nivel regional e internacional. Es en este aspecto que podemos observar cómo la experiencia Europea lleva la delantera en cuanto a PP e integración regional.

Además de lo que hemos dicho una de las características centrales de la actual PP es su relación con el conocimiento. En la sociedad del conocimiento éste es un factor de producción cada vez más decisivo pero además ello refuerza su poder. Nada más cierto que el conocimiento es poder pero también hay un poder del conocimiento en materia de PP en la sociedad globalizada actual.

Precisamente porque la PP es política y es poder ella está entonces ubicada en una instancia privilegiada en la gestión de conocimientos para definir, guiar e implementar adecuados cursos de acción en la sociedad actual. Pero en la sociedad del conocimiento se impone el poder del conocimiento en la definición de una agenda de PP.

Todo ello implica que en la actualidad la PP se ha profesionalizado. El rol y peso de los profesionales para la definición de agendas de política para la atención a problemas económicos y societales, y el afianzamiento de los Think Tanks, son decisivos en los procesos de formulación de modernas bases para la definición de PP.

Como disciplina o como ejercicio profesional la PP este último tiempo ha estado en ALC basada en métodos analíticos (principalmente económicos y cuantitativos, aunque no exclusivamente) lo cual lleva a Aguilar a 
afirmar para México algo que podría generalizarse a todo el continente, la $\mathrm{PP}$, “fue un elemento esencial y representativo del nuevo ambiente de cambio político y administrativo que se ha vivido en los últimos veinte años, ambiente que contribuyó además a generar y sostener tanto discursiva como prácticamente” (Aguilar 2004).

Rigurosidad en el análisis económico por una parte y jurídicoorganizacional por otra. Todo ello centrado en los procesos de formación, elaboración y toma de decisiones. De esta manera la nueva manera de ver a la PP la tendió a distinguir de la administración pública clásica dedicada más bien a la gestión eficiente en procesos post-decisionales.

Procesos de Reforma del Estado y de modernización de la gestión pública se han dado lugar en casi todos los países de la región (Tomassini, 1994). Estos procesos han generalizado este nuevo carácter de la PP. En este marco las PP que se elaboran hoy son "basadas en la evidencia” y por ende mucho menos ideologizadas como lo fueran durante el siglo XX. En las argumentaciones de la PP de hoy se da una simbiosis con las evidencias, lo cual permite comprender la vocación persuasiva de las políticas públicas (Goodin, 2006) e “imprime un nuevo carácter en la gobernanza que demanda nuevos estilos para hacer las políticas, de tal forma que maximicen los diversos esfuerzos de consulta, persuasión, deliberación y coordinación”. (Valenti y Flores 2009: 185)

De acuerdo a esta nueva realidad y dado que la PP está más basada en la profesionalización, su lógica se ha tornado mas pragmática lo cual inclina la balanza en su formulación y orientación hacia en enfoque tecnocrático (Joignant y Güell, 2011). La racionalidad de las PP se centra en el rendimiento eficiente. Se rechazan las visiones ideológicas, y el pragmatismo como punto de partida favorece las redes que se basan en la demostración y el convencimiento basado en conocimiento experto.

Estudios sobre hacedores de políticas hace un tiempo llegaron a la conclusión de que el empleo de las ciencias sociales, es decir del aporte del conocimiento académico, por parte de funcionarios de nivel superior es insuficiente. "La subutilización del conocimiento se debe en gran parte el interés de los policymakers en soluciones inmediatas y tangibles de los problemas reales”. (Valenti y Flores 2009: 183).

Anteriormente los policymakers estaban más influidos por las fuentes no profesionales de la información; ahora la agenda de las políticas públicas comenzó a poner el énfasis en la importancia estratégica de la evidencia, concebida ésta como punto de articulación entre información, conocimiento y aprendizaje.

Se trata entonces de mejorar la PP tomando en consideración los aportes que pueda hacer el mundo académico, el mundo de la ciencia y de los que investigan e innovan en los laboratorios y centros de investiga- 
ción. Esta necesidad de mejorar las PP se hace más necesaria en el contexto de los desafíos ambientales. Las políticas existentes y los incentivos de mercado han contribuido al problema de la mala asignación del capital ya que permiten a las empresas gestionar las externalidades sociales y ambientales, sin rendir cuentas y sin control. "Los mercados sin restricciones no están destinados a resolver los problemas sociales" (UNEP, 2011: 2). Hay una necesidad de mejorar las políticas públicas, incluyendo la fijación de precios y medidas reglamentarias, para cambiar el incentivo perverso del mercado que impulsa a esta mala asignación del capital y a ignorar las externalidades sociales y ambientales.

En materia de sustentabilidad y del área energética en particular las PP tiene todavía mucho que avanzar para impulsar la transición hacia nuevos paradigmas energéticos y de sustentabilidad ambiental.

Garantizar la sostenibilidad ambiental (séptimo Objetivo de Desarrollo del Milenio) requiere conseguir patrones de desarrollo sostenible y conservar la capacidad de producción de los ecosistemas naturales para las generaciones futuras. A su vez, ambos esfuerzos deben ir acompañados de una serie de políticas encaminadas a paliar los daños al medio ambiente y mejorar la gestión de los ecosistemas.

Este desafío presenta dos dimensiones: por un lado, hacer frente a la escasez de recursos naturales para los pobres del mundo y por otro, paliar los daños al medio ambiente derivados del alto consumo de las personas ricas (PNUD, 2003). Todos estos desafíos deben ser debidamente enfrentados por la PP en ALC.

\section{Relación mundo académico-políticas públicas: lo real y lo ideal}

Para nadie es un secreto que la actual relación entre el mundo de la academia y el mundo de las PP es problemática y adolece de déficits que muchos ignoran y otros tantos buscan remediar. Como se muestra en el diagrama la relación ideal entre estos mundos es por la vía del aporte que puede hacer el mundo académico a las PP en términos de retroalimentación de conocimientos sistemáticos, científicos, adecuados y pertinentes. No estamos afirmando que siempre que la academia aporta a la PP lo hace con este tipo de conocimientos, estamos afirmando que ese es el tipo de conocimiento que es requerido y que se le demanda, lo cual platea una exigencia al mundo académico. Los académicos deben entonces revisar y evaluar en qué medida se está aportando al mundo de las PP con este tipo de conocimientos. 


\section{Diagrama $N^{\circ} 1$ : Relaciones actuales entre el mundo académico y el mundo de las políticas públicas en la perspectiva del desarrollo humano y sustentable.}

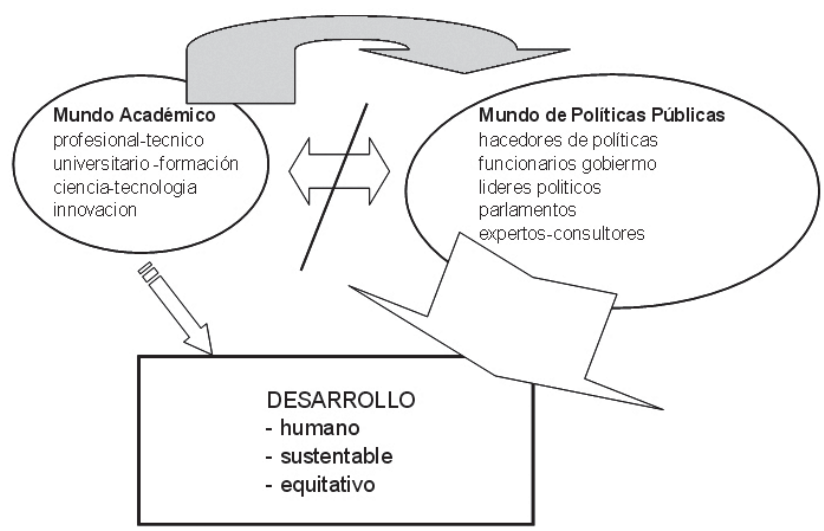

Fuente: elaboración del autor.

Si los profesionales y técnicos especializados, tanto como los científicos, y los centros de tecnología e innovación se encuentran en lo que llamamos en el mundo del conocimiento académico, no debemos olvidar que también resulta de gran relevancia para el aporte que se puede hacer a la PP, la función docente del mundo académico. No sólo porque las universidades y centros académicos forman futuros profesionales que constituirá el capital humano futuro de las PP sino porque desde la academia hay tareas innegables de contribución a elevar las capacidades y competencias del mundo de las PP.

Las propias instituciones académicas tienen sus propios programas de intervención en el mundo social y público (muchas universidades en el continente, más allá de sus programas de extensión cultural, cuentan con programas de salud, de educación, de superación de la pobreza, de combate a las drogas y la violencia, de ordenamiento territorial, de desarrollo social, de género, de desarrollo indígena, etc.). (Thorn y Soo, 2006; Parker, Muñoz, 2012)

De esta manera las universidades contribuyen indirectamente a la PP. Pero los hacedores de políticas, los funcionarios de gobierno, los expertos, técnicos y consultores de PP formados inicialmente en círculos académicos, en el desarrollo de sus procesos de formulación, implementación y evaluación de PP tienden a autonomizarse y a formar grupos cuyas interacciones sociales son más bien cerradas y autorreferentes, salvo sectores privilegiados del mundo de la academia ${ }^{7}$. 
El otro grupo que interviene en la PP los políticos y parlamentarios, a cargo de los ministerios y organismos planificadores del gobierno o los legisladores, generalmente operan sobre la base de conocimientos que son extraídos de su propia formación profesional y académica (que no siempre la tienen de calidad) y/o de órganos consultores (sus propios lobbies, expertos o entidades expertas como las Bibliotecas del Congreso).

Pero la interacción entre este mundo político y el mundo de la academia es puntual y ocasional, cuando ella se da, con ocasión de seminarios o encuentros. No habiendo lazos institucionales -que no habrá razón para ello- no existe tampoco mucho interés de parte de los políticos y legisladores de invertir tiempo en actividades académicas que se considera que obedecen a criterios alejados de la política, como tampoco de parte del mundo académico hay interés en verse involucrados en actividades que se consideran contingentes y desprestigiadas como la política.

Salvo grupos de académicos que por sus opciones políticas tienen mayor sensibilidad social y participan en actividades que directa o indirectamente inciden en la PP, la mayor parte de este mundo está más bien concentrado en sus actividades en la academia, formando profesionales, o haciendo investigación básica.

El resultado de todo este panorama que hemos esbozado es que los grupos de profesionales y ciencias que tienen la mayor incidencia en la elaboración, implementación y evaluación de la PP son los economistas, administradores públicos, ingenieros industriales y un conjunto de profesionales que se les asimilan. En materia de salud por ejemplo muchos médicos deben capacitarse en administración pública para incidir en PP; en materia educacional si los encargados de la política no cuentan con economistas expertos, sus programas arriesgan ser altamente cuestionados; en materia de previsión social son también los ingenieros comerciales e industriales los que intervienen; en materia de superación de la pobreza si hay sociólogos o geógrafos éstos deben someterse a la lógica de la gestión eficiente, etc. Sobre el privilegio de los economistas en la formulación de la PP en ALC ya se ha dicho bastante (Silva, 1996; Montecinos 1997; Estrada y PuelloSocarras, 2005; Ossandón, 2011).

Las definiciones de las orientaciones que han guiado a la PP durante muchos años ha estado centrada en un conjunto de acentos vinculados mucho más a la gestión y por ende a la intervención de los técnicos prevaleciendo una visión tecnocrática - más que a la definición adecuada de los problemas que se debe abordar y por ello con el concurso de los académicos y los científicos.

El centro de interés de “mejorar la gestión” (Lahera, 1993) a estado predominando por lo que resulta natural la proximidad de grupos como los técnicos o economistas tecnócratas, que a su vez son supervigilados por los políticos que tienen el mayor interés en que las políticas públicas resulten eficaces - o al menos que tengan visos de eficacia y productividad - a 
fin de asegurar su continuidad en el poder y obtener el beneficio de sus electores. No es que los políticos sólo actúen en beneficio de sus intereses, pueden estar bien inspirados en querer beneficiar a la población, pero en plazos y con efectos que están acotados a los períodos gubernamentales y parlamentarios.

\section{Esquema de proximidad de grupos involucrados en la PP:}

\begin{tabular}{|c|c|}
\hline Próximos & Lejanos \\
\hline Técnicos & Académicos \\
\hline Políticos & Científicos \\
\hline
\end{tabular}

Fuente: elaboración del autor.

Las políticas públicas que tienen éxito en lo sustancial son aquellas que pueden mantener los esfuerzos en un período prolongado y se enfocan a la solución de problemas de tipo estructural y/o de largo plazo, como por ejemplo la superación de la pobreza o los temas ambientales. Generalmente el mundo académico tiene los conocimientos y las herramientas para enfocarse hacia la solución de los problemas de largo plazo pero ello les hace ser de poca utilidad a los programas de políticas que deben rendir sus frutos en plazos acotados. Este dilema debiera ser enfrentado y superado en un nuevo tipo de dialogo entre mundo académico y mundo de las políticas públicas en nuestra región.

Un ejemplo de la relación real que es privilegiada entre el mundo de los consultores-profesionales y al mundo de las políticas públicas en ALC es el informe emanado de la CEPAL sobre Modelaciones para la PP en ALC (de Miguel et al, 2010).

Los autores y en particular este informe, propone instrumentos de modelización de la PP basados en la ciencia económica. La propuesta de generar una red de modeladores sobre base del Equilibrio Computable Glo$\mathrm{bal}^{8}$ apunta a facilitar el intercambio de hallazgos analíticos, técnicas y datos, entre la comunidad académica y científica lo que redundará en servir mejor a los policymakers en la formulación de políticas. La propuesta es valiosa, científicamente fundada y de proyecciones innegables.

Pero se trata de un enfoque acotado a cierto tipo de dimensiones de la problemática del desarrollo. El riesgo es que se tome a este tipo de enfo- 
ques como los de mayor credibilidad y validez por lo que se imponen como hegemónicos en la formulación de políticas ${ }^{9}$, dejando en un segundo plano enfoques que debieran ser o complementarios o alternativos y que consideran la realidad en términos de modelos más complejos, multidimencionales y multiescala. Por cierto enfoques como el mencionado de la CEPAL, siendo válidos, al presentarse como los de mayor legitimidad para servir a las PP. no propenden a una interacción adecuada entre mundo académico y mundo de las PP ya que al privilegiar modelos econométricos, prioriza a la economía como ciencia y no posibilita la interacción con otros grupos de la academia que pueden aportar dimensiones tecnológicas, culturales, sociales, ambientales y deja sentada la representación social de la que la mayor validez la tiene a ciencia economía en la interacción con los policymakers.

Un buen ejemplo de adecuada relación entre mundo de la academia y mundo de las políticas públicas lo encontramos en el Informe sobre la Transición Mundial y Europea en energía y ambiente, emanado de la Comisión Europea, el año 2011 (Château y Rossetti, 2011) y que recoge el resultado de programas de investigación multinacionales, multidisciplinarios y multinivel realizados en Europa desde 2001 sobre "Modelos de muy largo plazo Energía-Ambiente" (Proyect VLEEM) y "Pasos hacia la Transición de Carbono" (Proyecto PACT $)^{10}$. Se trata de estudios desarrollados en el marco de los programas europeos de investigación que combinan en un mismo y complejo proceso, investigación básica, investigación aplicada, innovación y difusión y que reciben el aporte de diversos centros académicos nacionales e internacionales y de distintas disciplinas y especialidades, en este caso de la sociología, la economía, la tecnología, varias ciencias naturales y la política. Estudios que aportan conocimientos válidos pertinentes a la PP sobre base de enfoques teóricos y metodológicos innovadores y que posibilitan el análisis científico de escenarios presentes y futuros por lo que consiste en un valioso insumo para la PP.

Una experiencia similar estamos desarrollando enfocada hacia la Gobernanza Ambiental en ALC a través del proyecto ENGOV, que incluye a centros de investigación de cuatro países europeos y de cinco países latinoamericanos ${ }^{11}$.

Esto está mostrado que un nuevo tipo de relación entre el mundo de la academia y el mundo de la PP es posible en la perspectiva de obtener una mejor comprensión de los desafíos de diseñar mejores políticas, y en particular con relación a la crisis ambiental, el CC y la gobernanza ambienta hacia la sustentabilidad.

\section{Por un nuevo paradigma de relación del mundo académico y el mundo de las políticas públicas}

El desafío de nuestros gobiernos frente a los retos de la interdependencia de la globalización, la nueva economía en la sociedad de la informa- 
ción, las exigencias de un orden internacional y nacional exigente y de sociedades diferenciadas, con demandas crecientes, donde persisten los problemas de la desigualdad y pobreza y aquellos derivados del medio ambiente, será recuperar una mirada estratégica, y constituirse en gobiernos eficaces en el tratamiento de los problemas públicos y con calidad y pertinencia gerencial e institucional. El mundo académico, en la medida en que esté dispuesto a bajar del altar de la ciencia en que se encuentra en muchos casos ensimismado -sobre la base de una reforma interna (Leher, 2010)- , podrá contribuir de manera eficiente y pertinente a elevar esa calidad, esa pertinencia, y esa eficiencia en la promoción de los cambios en la PP lo cual hará viable avanzar hacia una sociedad más sustentable y justa.

La intervención colaborativa del mundo académico apunta precisamente a la necesidad de incrementar un conocimiento de calidad para las políticas públicas. En efecto, las negociaciones comerciales en el comercio mundial, la erradicación de la pobreza y la desigualdad, la adaptación al CC y las nuevas políticas urbanas sustentables son claros ejemplos de políticas complejas con múltiples efectos directos e indirectos, así como consecuencias económicas, sociales, culturales y ambientales que son difíciles de evaluar, en ausencia de sofisticados instrumentos analíticos.

Pero es precisamente en materia de desarrollo sustentable y en la transición hacia una economía y sociedad verde que se requieren nuevos conocimientos (UNEP, 2011), análisis, indicadores, formas innovadoras de medición y evaluación, nuevos diagnósticos y planes. El incremento de la gobernanza ambiental, de marcos regulatorios y de inversión, el cambio hacia un modo de producción más limpio, el cambio en los patrones de consumo, la innovación en tecnologías verdes, las nuevas formas de planificación urbana, el incremento en capacidades y entrenamiento, el estudio del capital natural y la biodiversidad, etc. requerirán del concurso del conocimiento aportado por el mundo académico desde hoy y cada vez más en el futuro (Wesselink et al 2013).

El mundo de la academia tiene la responsabilidad de aportar dichos conocimientos que estarán retroalimentando a los tomadores de decisiones en la PP. A su vez los tomadores de decisiones y hacedores de PP tienen la responsabilidad de incrementar la calidad técnica, científica e integral de sus bases de conocimientos a fin de que sus políticas apunten a la complejidad de los problemas y sirvan de manera eficaz y comprensivamente a resolverlos en función de un desarrollo integral y sustentable.

Pero esta colaboración academia - políticas públicas se hace y debe hacerse en el marco de los procesos de cooperación e integración. Dadas las asimetrías de información y los fracasos en la coordinación que generalmente afectan a políticas públicas de calidad, las instituciones multilaterales de desarrollo tienen la oportunidad de apoyar a sus países miembros en la evaluación de las políticas que requieren un análisis complejo de su trasfondo (background), sobre todo cuando sus efectos se extienden más allá de la frontera de un país determinado. 
El desarrollo y la difusión de conocimientos de vanguardia y de herramientas analíticas deben por lo tanto, ser perseguidos como bienes públicos regionales (de Miguel et al., 2010: 13)

En ALC precisamente hemos presenciado, a diferencia de lo que percibimos como interesante experiencia en la UE, un déficit de cooperación en conocimientos que sostengan y eleven el nivel de las PP. En ALC falta aún conformar una apropiación de conceptos, así como identificar diversas problemáticas comunes que han impedido a la región establecer canales de cooperación más productivos. Falta además profundizar en la conformación de propuestas de solución así como generar escuelas de pensamiento propias para el intercambio de conocimiento.

Si es cierto que esfuerzos recientes buscan incorporar reflexiones sobre PP hacia el entendimiento de las circunstancias de cada país, aún se siguen incorporando modelos exitosos sin una reflexión más detallada de las probabilidades de éxito y la adecuación hacia los contextos sociales particulares. (Valenti y Flores 2009: 187).

\section{Diagrama Nº 2: Relación real e ideal entre las Políticas Públicas y laAcademia:}

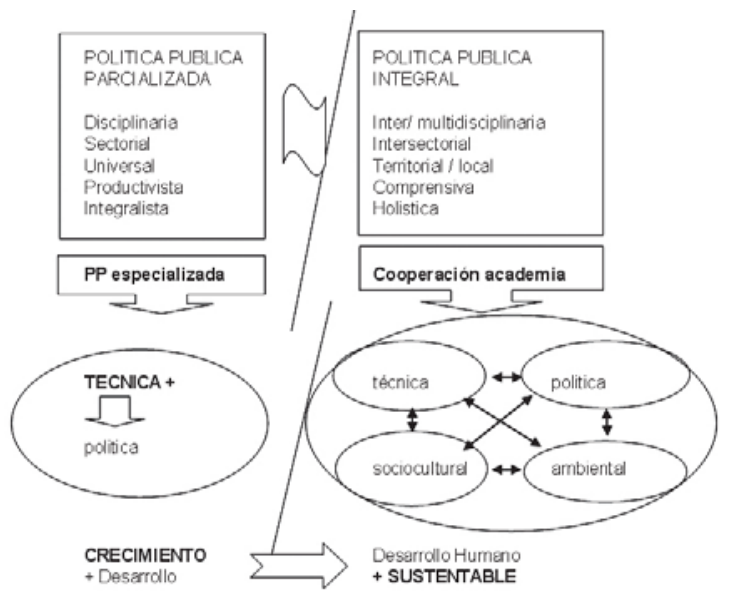

Fuente: elaboración del autor.

Para que la relación entre la academia y las PP sea fructífera en esta colaboración que apunte a elevar la calidad del conocimiento deberíamos avanzar hacia un cambio de paradigma desde el que está presente en la actualidad hacia uno que debería asumir los desafíos del auténtico desarrollo integral y sustentable. 
Como se observa en el diagrama la PP en la actualidad en la mayoría de los casos se desarrolla en ALC como una disciplina y un proceso especializado sobre la base de criterios de planificación, fundamentación e implementación de tipo universalista, basado en enfoques sectoriales, con objetivos de logro (enfocados hacia la productividad) y con una mirada global a los problemas y necesidades que apunta. El énfasis está puesto en objetivos de rendimiento técnico (eficacia y eficiencia) buscando consecuencias políticas inmediatas. No cabe duda que muchas de las políticas de las últimas décadas han sido parcialmente exitosas en la obtención de las metas del crecimiento y la mejora en las condiciones y bienestar de la población lo cual lleva, por lo demás a los europeos en estos días a resaltar las lecciones positivas de la experiencia latinoamericana frente a la crisis que se vive en el viejo continente.

Pero, como lo muestra la segunda sección de nuestro diagrama, si de veras se quiere avanzar hacia un desarrollo humano y sustentable en nuestros países, la magnitud de los cambios que se requieren demanda una reingeniería de las actuales PP sobre la base de enfoques que se han estado probando como parcialmente exitosos en algunos casos puntuales. Se trata de avanzar hacia objetivos de PP que estén centrados en procesos de autodesarrollo a nivel de localidades y regiones, elevando las propias capacidades de las comunidades para asumir sus problemas y redefiniendo un pacto programático entre el estado central y las instancias subnacionales a fin de avanzar en políticas que sean no sólo eficaces y eficientes, sino también pertinentes y sustentables en función de procesos de ecodesarrollo a nivel comunitario y regional.

Por experiencia propia ${ }^{12}$ sabemos que la colaboración de la academia en generar políticas públicas desde perspectivas inter y multidisciplinarias, con carácter intersectorial y con enfoques territoriales y descentralizados, sobre base de procesos holísticos, multicriterio, multiescala, multidimencionales, y con enfoques generales comprensivos (que integran criterios “duros” y "blandos”, económicos-productivos y socioculturalesambientales) es no sólo posible sino necesaria.

De esta manera el nuevo paradigma de PP que se genera desde la finalidad del auténtico desarrollo humano y sustentable viene a posibilitar la interacción y debida consideración de las cuatro macrodimensiones de los procesos de gestación, formación, toma de decisiones, implementación y evaluación de las PP. Nos referimos a las macro dimensiones: a) Técnica y tecnológica; b) Política; c) Sociocultural y c) Ambiental. Una de ellas que esté ausente o que sea considerada marginalmente y todo el edificio de la sustentabilidad amenaza con derrumbarse.

Adicionalmente una adecuada relación dialógica entre academia ciencia-tecnología de una parte y mundo de las PP de otra tiene la ventaja de generar condiciones para evitar el riesgo de la influencia tecnocrática en la PP, así como el riesgo del cientificismo teórico en la academia. 
La modernización del Estado requiere que los cambios realizados en los niveles institucionales, sectoriales, sistemas y de servicios operen según lo previsto y den una respuesta adecuada a los problemas que presenta la sociedad. Las Políticas Públicas se transforman en una herramienta crítica para el éxito de los cambios demandados por la sociedad. Además se debe asegurar la participación social en la formulación y aplicación de las políticas públicas.

El diálogo y la cooperación entre la academia y la PP corre el riesgo de reforzar el predominio de criterios técnicos - incluso tecnocráticos- si no se da en el marco de procesos participativos donde la voz de los ciudadanos y en especial de aquellos que son los necesitados, así como los involucrados y/o beneficiados por los programas públicos no es toma en consideración en forma real (Braun, 2010; ENGOV, 2013). Esto requiere de parte de los académicos agudizar una sensibilidad que no siempre está presente en el mundo de la academia y de la investigación en laboratorio: escuchar a los simples ciudadanos acerca de sus demandas, críticas, necesidades y sueños, y abrir los espacios deliberativos acerca de las reformas verdes generando siempre instancias democráticas (Hysing, 2013) de diálogo y aprendizaje común.

Requiere también que la PP se estructure sobre el diálogo con la academia pero teniendo siempre en cuenta a los ciudadanos que constituyen la tercera base de apoyo para que toda PP sea democrática y viable en términos de gobernanza futura. En materia ambiental los procesos de Evaluación de Impacto Ambiental y de Evaluación Ambiental Estratégica, debieran contemplar no sólo una “consulta” a las comunidades afectadas localmente por los proyectos, sino que debiera contemplar una participación real de las comunidades y movimientos ciudadanos, que a partir del empoderamiento de su rol de ciudadanos ambientales, puedan prestar su compromiso con esos mismos proyectos en tanto y en cuanto aseguren un impacto ambiental mitigado o compensado y formas y procedimientos más limpios, con menor huellas de carbono y ecológicamente sustentables.

Como recuerda Tomassini (2004:162) una de las condiciones básicas para la gobernabilidad democrática reside en la calidad de las políticas públicas con que el sistema enfrenta la demanda de sus ciudadanos.

En la generación, implementación y desarrollo de un nuevo pacto entre el mundo de la academia y el de las políticas públicas, subordinado como hemos propuesto hacia las tareas de cambio hacia la sustentabilidad, la integración regional en el marco de la CELAC parece ser un instrumento de primer orden.

Efectivamente la emergencia de una nueva agenda internacional, en donde crecientemente se ubican temas globales, obliga a la región latinoamericana a diseñar respuestas coordinadas entre los Estados, y entre estos y los actores no estatales, para enfrentar estos nuevos procesos (Rojas, 2011). Este espacio de dialogo entre el mundo de la PP y el mundo de la 
academia encontrará su funcionalidad precisamente en esa perspectiva.

A propósito de la I Cumbre CELAC-UE desarrollada en enero de 2013, creemos que la cooperación UE y el CEALC en materia de PP es imprescindible y en dicha cooperación el mundo de la academia tiene un evidente y necesario rol que jugar.

Dada la experiencia europea en materia de PP que apuntan a procesos de desarrollo sustentable, experiencia más avanzada que en ALC, la UE podría prestar su cooperación en términos de compartir conocimientos y expertice en procesos de producción y difusión de conocimientos que contribuyan a incrementar presencia de la academia en PP. Ejemplos de ello es en lo relativo a patentes en Tecnologías Verdes, intercambios de experiencias sobre tecnología, normas y reglamentaciones en materia de biocombustibles, energía hidroeléctrica y energías renovables.

Asimismo en el marco de la CELAC debiera estimularse un espacio de intercambio de ALC en torno a la relación academia- PP para aprovechar experiencias diversas y replicables. Este espacio sería referente del espacio Europeo homólogo. La emergencia de una nueva agenda internacional, en donde crecientemente se ubican temas globales, obliga a la región latinoamericana a diseñar respuestas coordinadas entre los Estados, y entre estos y los actores no estatales, para enfrentar estos nuevos procesos y sus consecuencias nacionales y regionales.

Pero también ALC podría ofrecer a la UE su propia experiencia. En efecto, las experiencias y conocimientos locales de los países de AL podrían servir a los europeos para cotejar sus teorías y formulas de solución en PP y así incrementar y enriquecerse mutuamente.

En todo este intercambio, recopilación de experiencias exitosas de PP, prácticas de nuevos paradigmas de relación en cuanto a avances en sustentabilidad, la academia, con sus redes, sus instancias y sus potencialidades de intercambio, (y de constitución de un espacio de conocimientos en la RED o Foro Académico Eurolatinoamericano) puede ser una instancia fundamental para este cometido.

Pero esta propuesta, para que tanga proyecciones en el tiempo, requiere avanzar en alguna forma de institucionalización del intercambio. Para que la academia se abra a esta colaboración y las PP escuchen mas a la academia es necesario crear instancias de diálogo y ojala permanentes. Se deben, por ello crear mecanismos mas institucionalizados que permitan a la academia superar su aislacionismo y aportar a la PP, generando también los necesarios incentivos para volcar los conocimientos básicos y fundamentales al terreno de la aplicación, avanzando desde la ciencia básica a la ciencia aplicada y de allí hacia la PP.

Ello será responsabilidad de los organismos pertinentes (comisiones de ciencia y tecnología, programas de ciencia e innovación, etc.) como 
del mundo universitario (universidades y centros de la investigación).

Por su parte al mundo de la PP se le piden procedimientos y normativas, así como reacomodar sus patrones lógicos de planificación y gestión, a fin de establecer un dialogo e intercambio mas permanente intersectorial, interdisciplinario y de multinivel con el mundo de la academia para beneficiarse de sus conocimientos.

La responsabilidad para esta adecuación del mundo de las PP no sólo provendrá de los organismos especializados y técnicos en la formulación, análisis y evaluación de la PP, sino que fundamentalmente provendrá de los gobiernos y del mundo político. A fin de cuentas las tareas del auténtico desarrollo humano y sustentable, para las cuales la PP y la academia deben reinventarse, y volver a relacionarse, son de orden político. Los pueblos y naciones latinoamericanos y caribeños merecen ser conducidos por opciones políticas que les garanticen un mundo futuro -para ellos y las futuras generaciones- más equitativo, y ambientalmente sustentable. 


\section{Notas}

1 Doctor en Sociología, Profesor del Doctorado en Estudios Americanos, Instituto de Estudios Avanzados, Universidad de Santiago de Chile. Responsable del equipo chileno en el proyecto internacional europeo-latinoamericano ENGOV. "Gobernanza Ambiental en América Latina y el Caribe: Desarrollando Marcos para el Uso Sostenible y Equitativo de los Recursos Naturales”.

2 Este trabajo es una actualización de una ponencia presentada en la I Cumbre Académica (Santiago de Chile, 22 y 23 de enero de 2013) que precedió a la I Cumbre CELAC-UE (26-27 de enero de 2013) y a la I Cumbre de la CELAC (27 de enero de 2013) en Santiago de Chile.

3 La principal temática de esta I Cumbre de Jefes de Estado y Gobierno CELAC-UE fue "Alianza para un Desarrollo Sustentable: Promoviendo Inversiones de Calidad Social y Ambiental".

4 En América Latina se ha generado una política internacional más autónoma y se ha visto el ascenso de gobiernos de centro-izquierda. Sus objetivos son la búsqueda de una mayor soberanía, y la puesta en práctica de modelos de desarrollo, endógenos y alternativos, adoptando conceptos como el "buen vivir" y promoviendo una mayor participación ciudadana. Incluso los gobiernos que favorecen modelos de mercado también han incorporado y desarrollado de manera muy importante políticas públicas con orientación social. Sin embargo, los gobiernos de la región con distintas orientaciones políticas concuerdan en que deben basar sus estrategias de crecimiento en la promoción de las inversiones extranjeras o bien en la inversión estatal en la explotación de recursos naturales, principalmente en el área extractiva y agroalimentaria, en función del mercado internacional retornando a lo que se ha llamado un modelo de “reprimarización” de sus economías exportadoras. Lo cual es más evidente en América del Sur. (ver Hogenboom y Fernández, 2009; CEPAL, 2012; ENGOV, 2013).

5 Sobre los desafíos ambientales de la energía y el agua en América Latina ver nuestro análisis en Parker (2014).

6 Programa de Investigación colaborativa del FP7 de la Comisión Europea que involucra una iniciativa de investigación conjunta Europa- América Latina (proyecto ENGOV) sobre “Gobernanza Ambiental en América Latina y el Caribe: Desarrollando Marcos para el Uso Sostenible y Equitativo de los Recursos

${ }^{7}$ En el caso chileno que conocemos de cerca, por ejemplo dos o tres instancias de la Universidad de Chile como el Dpto. de Ingeniería Industrial y el Centro de Análisis de Políticas Publicas y algunos Think Tanks independientes tienen cierta interacción con el mundo de las PP. Durante el gobierno de Sebastián Piñera los centros de la PUC tomaron el relevo. Pero el grueso del mundo académico y universitario se ha relacionado escasa o nulamente con el mundo de las PP.

8 CGE: computable general equilibrium, en original en inglés en el texto.

${ }^{9}$ El trabajo referido entiende que los modelos cuantitativos como el CGE tienen limitaciones pero concluyen que se trata de la única "técnica que provee una manera global e interrelacionada para captar los efectos económicos amplios de una reforma 
política” (de Miguel et al, 2010: 17).

10 Very Long Term Energy Environment Model, en: www.VLEEM.org; y Pathways for Carbon Transitions en: www.pact-carbon-transition.org .

11 El mencionado proyecto colaborativo entre investigadores latinoamericanos y europeos se enfoca en iniciativas recientes de gobernanza ambiental en la región, con el fin de analizar los obstáculos y oportunidades para que los sistemas de producción sostenibles puedan generar tanto desarrollo económico como una distribución más equitativa de los beneficios. El proyecto explora alternativas de innovación institucional y aproximaciones inclusivas al uso de los recursos naturales que contribuyan a reducir la pobreza, la inequidad y la degradación ambiental en América Latina y el Caribe.

ENGOV es un proyecto de investigación multidisciplinario que aplica diversos métodos cualitativos y cuantitativos de las ciencias ambientales, la economía, la sociología, la ciencia política, la antropología y la historia. Participan centros de investigación de Holanda, Francia, España y Noruega, así como Argentina, Brasil, Chile, Ecuador y México. Los principales países donde se desarrollan investigaciones empíricas son Argentina, Bolivia, Brasil, Chile, Colombia, Ecuador, El Salvador, Guatemala y México. El autor forma parte del Comité Científico de esta iniciativa. Ver www.engov.eu.

${ }^{12}$ Nos ha tocado trabajar la última década y media desde el mundo académico apoyando y evaluando políticas públicas en desarrollo social y superación de la pobreza, desarrollo indígena, políticas de juventud, educación intercultural, políticas de medidas penales alternativas y políticas de promoción del turismo en Chile. 


\section{Bibliografía}

Aguilar Villanueva, Luis F. (2004), “Recepción y desarrollo de la disciplina de PP en México. Un estudio introductorio”, Sociológica, Año 19, N54, 15-37.

Arriagada, Irma (2006), “Cambios de las políticas sociales: políticas de género y familia”, Serie Políticas Sociales No 119, CEPAL, División de Desarrollo Social. Santiago de Chile.

Baud, M., de Castro, F., y Hogenboom, B. (2011), “Environmental Governance in Latin America: Towards an Integrative Research Agenda (Explorations)", European Review of Latin American and Caribbean Studies 90, 79-88.

Banco Mundial (2010), Informe sobre Desarrollo Mundial 2010, Desarrollo y Cambio Climático, Panorama general un nuevo clima para el desarrollo. World Bank. Washington D.C.

Braun, R. (2010), “Social participation and climate change”. En Environment, Development and Sustainability, 12, 777-806.

Bull, B. (2010), “The Global Elite, Public-Private Partnerships and Multilateral Governance”. In Clapp, J. and Wilkinson R. (Eds). Global Governance, Poverty and Indequality, Routledge, 209-234. London.

Cashore, B. (2002), “Legitimacy and the Privatization of Environmental Governance: How Non-State Market-Driven (NSMD) Governance Systems Gain Rule-Making Authority”. Governance: An International Journal of Policy, Administration, and Institutions, 15 (4), 503-529.

CEPAL (2012), La Unión Europea y América latina y el Caribe: Inversiones para el crecimiento la inclusión social y la sostenibilidad ambiental, Naciones Unidas, CEPAL. Santiago de Chile.

Château, Bertrand y Rossetti di Valdalbero, Domenico (Eds.) (2011), World and European Energy and Environment Transition Outlook WETO-T, Directorate-General for Research and Innovation, Socio-economic Sciences and Humanities, European Commission. Bruselas.

Clapp, J. (1998), “The Privatization of Global Environmental Governance: ISO 14000 and the Developing World”. Global Governance, 4 (3), 295316.

Consejo de la UE (2010), "Hacia una nueva etapa en la asociación birregional: la innovación y la tecnología en favor del desarrollo sostenible y de la inclusión social” Declaración de Madrid, VI Cumbre UE-ALC, 18 de mayo de 2010. Madrid. 
De Miguel, Carlos, José Durán Lima, Paolo Giordano, Julio Guzmán, Andrés Schuschny, Masakazu Watanuki (Eds) (2010), Modeling Public Policies in Latin America and the Caribbean, ECLAC, IDB, September 2010, Santiago, Chile.

Dye, Thomas R. (1992), Understanding Public Policy, Prentice Hall. Englewood Cliff.

ENGOV (2013), La gobernanza ambiental de actividades extractivas en América Latina y el Caribe: la necesidad de incluir a las comunidades, European Policy Brief, ENGOV Policy Brief Nº1, Comisión Europea. Bruselas. Disponible en www.engov.eu.

Estrada Alvarez, Jairo; Jose Francisco Puello-Socarras, (2005), “Elites, intelectuales y tecnocracia Calidoscopio contemporáneo y fenómeno latinoamericano actual”, Colombia Internacional, Universidad de Los Andes, Julio - Diciembre, pp. 100 - 119, en: http://colombiainternacional.uniandes. edu.co/view.php/473/index.php?id=473

Etzkowitz, Henry y Leydesdorff, Loet (2000), “The dynamics of innovation: from National Systems and "Mode 2" to a Triple Helix of university-industry-government relations”, Research Policy, V. 29, 109123.

Ford, L.H. (2003), “Challenging Global Environmental Governance: Social Movement Agency and Global Civil Society”. Global Environmental Politics, 3 (2), 120-134.

Gomáriz Moraga, Enrique (2007), Sistema político y políticas públicas en América Latina, CLAD: Centro Latinoamericano de Adinistración para el Desarrollo, junio 2007, en: http://www.clad.org/portal/publicaciones-delclad/revista-clad-reforma-democracia/articulos/038-junio-2007/sistemapolitico-y-politicas-publicas-en-america-latina

Goodin, Robert et al (2006), “The Public and its Policies”, en The Oxford Handbook of Public Polcy, R. Goodin (cord), Oxford Uiversity Press, 3-35. Nueva York.

Gibbons, M., Limoges, C., Nowotny, H., Schwartzman, S., Scott, P. y Trow, M. (1994), The New Production of Knowledge: The Dynamics of Science and Research in Contemporary Societies, SAGE. Londres.

Hessels, Laurens K. y Van Lente, Harro (2010), “The mixed blessing of Mode 2 knowledge production”, Science, Technology \& Innovation Studies V. 6, (1), August, 65-69.

Hogenboom, B., Fernández, A.E. (2009), “The New Left and Mineral Politics: What's New?”. Revista Europea de Estudios Latinoamericanos y del Caribe, 87, 93-102. 
Hysing, Erik (2013), “Representative democracy, empowered experts, and citizen participation: visions of green governing”, Environmental Politics, Vol 22, Issue 6, pp. 955-974, DOI: 10.1080/09644016.2013.817760.

IEA (2013), World Energy Outlook Special Report, Redrawing the EnergyClimate Map, International Energy Agency. Paris, junio, disponible en: www.worldenergyoutlook.org.

Ídem (2012), World Energy Outlook 2012, Internacional Energy Agency. Paris, noviembre, disponible en: www.worldenergyoutlook.org.

IPCC (International Panel on Climate Change) (2007), Climate Change, Synthesis Report. IPCC, OMM, PNUMA. Geneva. http://www.ipcc.ch/ pdf/presentations/briefingbonn-2007-05/mitigation-substainabledevelopment.pdf.

Joignant A., Guell, P. (Eds) (2011), Notables, Tecnócratas y Mandarines, Elementos de sociología de las elites en Chile, (1990-2010). Ediciones Universidad Diego Portales. Santiago de Chile.

Lahera Parada, Eugenio (2002), Introducción a las Políticas Públicas, Ed. FCE. Santiago.

Ídem (1993), “Políticas Públicas, un enfoque integral”, en E. Lahera (ed), Como Mejorar la Gestión Pública, CIEPLAN, FLACSO, Santiago de Chile, Foro 90, 19-49.

Leher, R., (Comp.) (2010), Por una reforma radical de las universidades latinoamericanas, Clacso. HomoSapiens, Rosario, Argentina.

Montecinos, Verónica (1997), “Los economistas y las élites políticas en América Latina”, Estudios Internacionales, Vol. 30, No. 119-120 (1997).

Moreno, Marco (2009), “Proceso y tensiones en la formación de políticas”, en Curso Planificación y Gestión Estratégica para las Políticas Públicas, ILPES-AECID. Santa Cruz, Bolivia, 4-15 Mayo. Disponible en: www.cepal.org/ilpes/noticias/.../7/.../C14_Proceso_Formacion_PP.ppt

Ossandón, José (2011), “Economistas en la elite: entre tecnopolítica y tecnociencia”, en Joignant y Guell (eds), Notables, Tecnócratas y Mandarines, Elementos de sociología de las elites en Chile, (1990-2010). Ediciones Universidad Diego Portales, pp. 109- 126. Santiago de Chile.

Oviedo, Enrique (2009), Operacionalizacion de metas y objetivos de una política y su seguimiento, en V Curso Internacional de Capacitación en Prevención Comunitaria del Delito, 03 de julio, en http:// comunidadyprevencion.org/wp/docs/valpo_presentaciones/ Enrique\%20Oviedo-Operacionalizacion-Metodologias.pdf 
Paavola, J. (2007), “Institutions and environmental governance: A reconceptualization”. Ecological Economics, 63, 93-103.

Parker, C. (2011), “Global Warming, Elites And Energy In Latin America: The Chilean Case”, Journal of Environmental Science and Engineering, V. 5, No 12, December. 1702 - 1714.

Parker, C., Muñoz, J. (2012), “Elites universitarias y cambio climático”, Ambiente \& Sociedade, São Paulo XV (2) 195-218.

Parker, C (2014), "Patrones de consumo de energía y agua y desafíos ambientales en América Latina”, en Fernando Estensoro (ed), Diálogos Europeo Latinoamericanos de Ecología Política. Proyección del debate sobre medio ambiente y desarrollo en el siglo XXI, Ed. Universidad de Santiago, VRIDEI, Capitulo 9 (en prensa), Santiago de Chile.

PNUD (2003), Informe sobre Desarrollo Humano 2003, Los objetivos dedesarrollo del Milenio: un pacto entre las naciones para eliminar la pobreza, PNUD, PNUD - Mundi Prensa. Madrid.

Podestá Arzubiaga, Juan (2001), “Problematización de las políticas públicas desde la óptica regional”, Ultima década. [online]. 2001, V. 9, Nº.15, 163-175.

Poole, Richard E. (2012), "Global Governance and the Environment: Evaluating the Effectiveness of Global Governance in Tackling Contemporary Environmental Issues”, Student Pulse, The International Student Journal, Vol 4 Nº 6, pp. 3-31.

Ramírez Orrego, Ariel (2008), “Políticas públicas en época de cambio”, Curso de formación general, Estado y Política en el siglo XXI, Universidad de Chile, Vicerrectoría Académica, Semestre Otoño. Santiago.

Rojas Aravena, Francisco (2011), Nuevos instrumentos latinoamericanos para el multilateralismo y la integración, el caso de la CELAC, FLACSOSecretaría General, Costa Rica, en: www.flacso.org

Sachs, Jeffrey D. y Vial, Joaquin (2002), Competitividad y Crecimiento Económico en los Países Andinos y en América Latina, Proyecto Andino de Competitividad, Documento de Trabajo, Center for International Development, Harvard University. Cambridge, MA.

Samaniego, J. (2009), Cambio climático y desarrollo en América Latina y el Caribe: una reseña. CEPAL, GTZ, Naciones Unidas. Santiago de Chile.

Silva, Eduardo (1996), The state and capital in Chile: Business elites, technocrats, and market economics, Westview Press, Boulder, Colo.

Thorn, K; Soo, M. (2006), Latin American Universities and the Third 
Mission. Trends, Challenges and Policy Options, World Bank Policy Research Working Paper 4002, World Bank. Washington DC.

Tomassini, Luciano (2004), “La gobernabilidad en los tiempos del caos”, en FLACSO-CHILE, Chile 2003-2004 Los nuevos escenarios (inter) nacionales, FLACSO-Chile, 159-176. Santiago.

Ídem (1994), La Reforma del Estado y las Políticas Públicas. Centro de Análisis de Políticas Públicas, Universidad de Chile, Santiago.

UNDP (United Nations Development Programme) (2011)a, Human Development Report 2011,Sustainability and Equity: A Better Future for All. UNDP. New York.

UNEP (2011), Towards a Green Economy: Pathways to Sustainable Development and Poverty Eradication - A Synthesis for Policy Makers, UNEP, disponible en: www.unep.org/greeneconomy

Valenti Negrini, Giovanna y Flores Llanos, Ulises (2009), “Ciencias Sociales y Políticas Públicas”, Revista Mexicana de Sociología, 71, Num. Especial, 167-191.

Vergara, W. (ed) (2009) “Assessing the Potential Consequences of Climate Destabilization in Latin America”. LCR Sustainable Development Working Paper No. 32, The World Bank Latin America and the Caribbean Region Sustainable Development Department (LCSSD), Washington D.C.

Wallace, Arturo (2011), América Latina: no tan competitiva como debería, BBC - Mundo, Bogotá, 2 de diciembre, disponible en http://www.bbc.co.uk/ mundo/noticias/2011/12/111201_economia_competitividad _america_latina_colombia_aw.shtml 